\title{
Acral ischemia as a presenting manifestation of essential thrombocythemia
}

\author{
Maher Hanoun · Alexander Röth • \\ Ulrich Dührsen · Franz-Eduard Brock
}

Received: 1 June 2011/Revised: 30 July 2011 / Accepted: 2 August 2011/Published online: 20 August 2011

(c) The Japanese Society of Hematology 2011

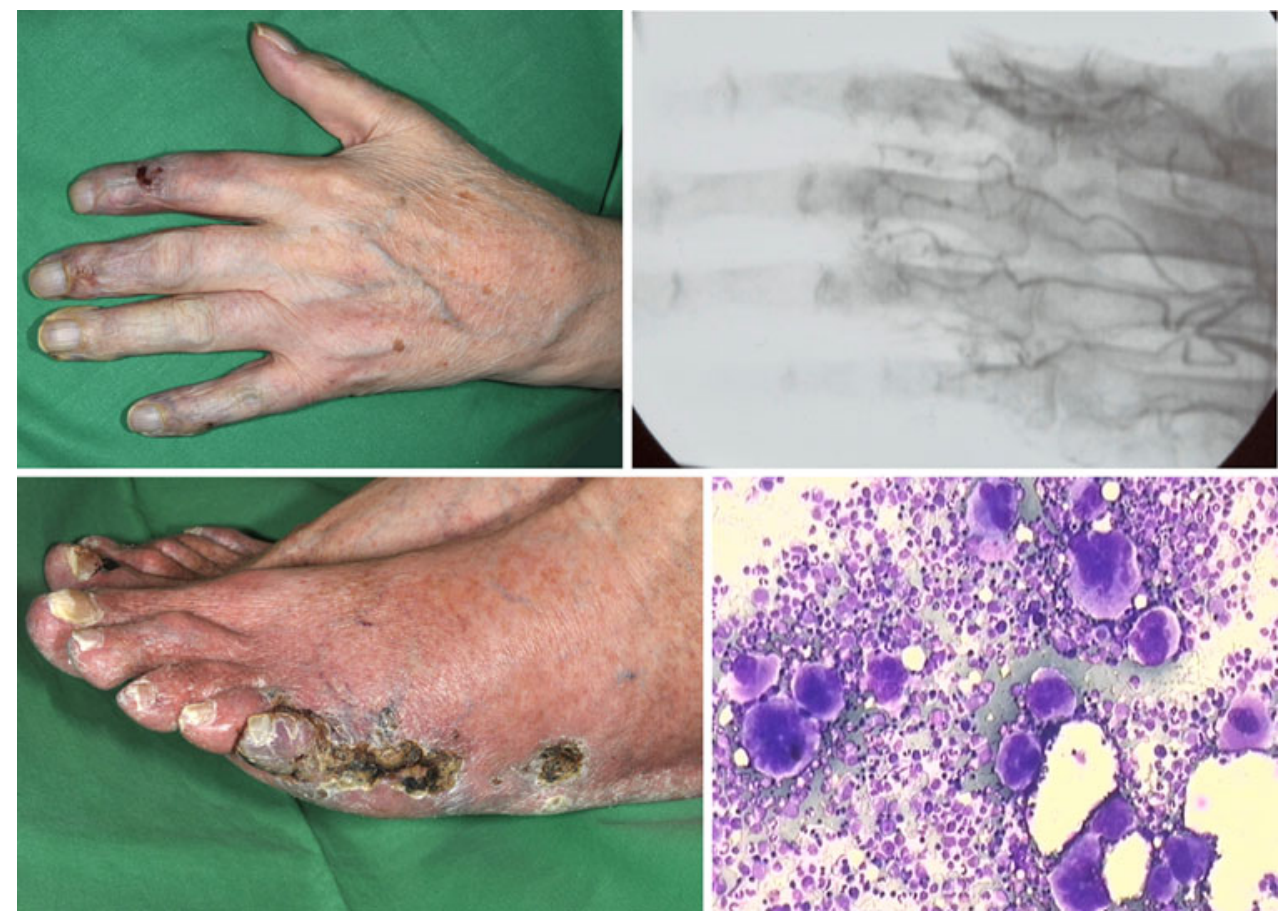

A 74-year-old male presented with a 6-week history of rapidly progressive acral ischemia. Clinical examination revealed livid discoloration of his toes and fingertips. All

\section{Hanoun · F.-E. Brock}

Department of Angiology, University Hospital,

University of Duisburg-Essen, Essen, Germany

\section{Hanoun $(\bowtie) \cdot$ A. Röth · U. Dührsen}

Department of Hematology, University Hospital,

University of Duisburg-Essen, Hufelandstr. 55,

45122 Essen, Germany

e-mail:maher.hanoun@uk-essen.de peripheral pulses were palpable, and ankle brachial pressure indices were normal. Laboratory tests revealed thrombocytosis of 710,000/ $\mu 1$ (normal range 140,000 $320,000 / \mu \mathrm{l}$ ), while red blood cells were within normal range and leukocytes were moderately elevated, indicating neutrophilia but otherwise normal differential blood count.

Angiography showed obliteration of all digital arteries (top right panel). There were no proximal stenoses or plaques. A cardiac source of embolism was excluded by transesophageal echocardiography. Despite intravenous and intraarterial administration of prostaglandin E1 and acetylsalicylic acid medication, the ischemic areas rapidly 
increased, with partial gangrene of the little toe of the patient's left foot and necroses of the fingertips of his left hand (top and bottom left panels). Bone marrow aspirate and biopsy met the diagnostic criteria for essential thrombocythemia (bottom right panel), which was further confirmed by demonstration of a JAK2 V617F mutation. Cytoreductive therapy with hydroxyurea was followed by significant clinical improvement with demarcation of the ischemic areas.

Essential thrombocythemia should always be considered in the differential diagnosis of acral ischemia. Rapid initiation of therapy with cytoreductive agents is crucial to prevent irreversible tissue damage. 